University of Wollongong

Research Online

Faculty of Engineering and Information

Faculty of Engineering and Information

Sciences - Papers: Part B

Sciences

2017

Towards Cost Reduction in Cloud-Based Workflow Management through

Data Replication

Fei Xie

University of Wollongong, fx439@uowmail.edu.au

Jun Yan

University of Wollongong, jyan@uow.edu.au

Jun Shen

University of Wollongong, jshen@uow.edu.au

Follow this and additional works at: https://ro.uow.edu.au/eispapers1

Part of the Engineering Commons, and the Science and Technology Studies Commons

Research Online is the open access institutional repository for the University of Wollongong. For further information contact the UOW Library: research-pubs@uow.edu.au 


\title{
Towards Cost Reduction in Cloud-Based Workflow Management through Data Replication
}

\author{
Keywords \\ workflow, towards, management, cost, data, replication, reduction, cloud-based \\ Disciplines \\ Engineering | Science and Technology Studies

\section{Publication Details}

Xie, F., Yan, J. \& Shen, J. (2017). Towards Cost Reduction in Cloud-Based Workflow Management through Data Replication. 5th International Conference on Advanced Cloud and Big Data (pp. 94-99). 


\title{
Towards Cost Reduction in Cloud-Based Workflow Management through Data Replication
}

\author{
Fei Xie, Jun Yan, Jun Shen \\ School of Computing and Information Technology \\ University of Wollongong \\ Wollongong, NSW, Australia \\ fx439@uowmail.edu.au, \{jyan,jshen\}@uow.edu.au
}

\begin{abstract}
In the cloud, data replication strategies have been adopted by many geographical data centers in order to improve data availability, decrease the access latency and reduce the data communication cost. In this paper, we propose a novel data replication strategy to reduce the cost of data storage and transfer for workflow applications. In our approach, we partition data storage space into two categories, and classify dataset types into three categories. We also develop a data replication algorithm in the buildtime stage with different determinant levels of data dependency, access frequency, storage capacities of data centers, and size of datasets. The case study shows that our approach can significantly decrease the total cost of data storage and transfer for workflow applications.
\end{abstract}

Keywords-access frequency, cloud computing, data replication, dataset dependency, workflow

\section{INTRODUCTION}

In recent years, cloud computing technologies constructs a novel approach for massive data processing and storage owing to its higher processing performance, its lower cost of infrastructure establishment and its higher interoperability [13]. Nowadays, many workflow applications adopt cloud computing technologies as the basis of run-time environment in order to obtain a great value from cloud. But there are still some challenging issues for deploying workflow applications in a cloud environment. Some datasets in the workflow may be very large and quite expensive to move around when one task in the workflow needs to process data from data centers in other locations [17]. Moving those data may be costly, inefficient and infeasible for those workflows. The past research has proposed different solutions to this problem. Specific data placement strategies can reduce data movement and save data transfer cost among data centers by placing data to appropriate data centers [17]. Further, data replication strategy can decrease the cost of data transfer because efficient replication of frequently used data can also reduce the data movement and cost [12].We argue that data replication is a multi-dimensional problem and multiple data attributes and environmental attributes need to be considered within an integrated approach. Hence, this research integrates access frequency, dataset dependency, storage capacity of data centers, and size of datasets together to develop the data replication strategy for each dataset.

Our data replication strategy is applicable at the build-time when initial data placement strategy is finished. We set three threshold parameters for dataset dependencies among datasets, access frequencies of datasets, and storage capacity of data centers. Dataset dependency among datasets and access frequency for each dataset are calculated as constraints of the dataset. At the same time, we use the threshold value of storage space to limit data replication to avoid overflow problems and ensure full task completion in the corresponding location. We also classify data types into three categories, fixed dataset, free-flexible dataset and constrained-flexible dataset, to construct a mapping between datasets and each data center. By adopting our strategy, we attempts to further decrease data movement and data transfer cost.

The remainder of the paper is organized as follows. Section II presents the related work. Section III describes the system model of our work. Section IV presents the detailed steps of our basic data replication strategy and our build-time data replication algorithm. Section V demonstrates the case study and relevant discussions to evaluate our data replication strategy. Finally, Section VI addresses our conclusions and future work.

\section{RELATED WORK}

In cloud environments, data replication strategy is the strategy of creating multiple data copies and storing the copies in multiple sites [12]. Data replication can help users save response time when tasks are being processed in the cloud, and improve the data availability [16] and reliability [6,7]. It can also save the bandwidth consumption [2], decrease the data access latency [5,9], and reduce the data transfer amounts, time and costs [4]. Appropriate data replication strategy is complicated in a large scale cloud system [11]. Data replication strategy can further be classified into two mechanism types, static and dynamic [12]. In [10], a Multi-objective Optimized Replication Management strategy is proposed to balance the trade-off among five optimization objectives, including the mean service time, mean file unavailability, load variance mean access latency and energy consumption, to make a near optimal solution for data replication. In [3], a Dynamic Cost-aware Rereplication and Re-balancing strategy (DCR2S) is proposed for knapsack problems in three phases, by determining suitable data file and numbers of data file replicas to replicate to an appropriate location, and determining additional required replication for satisfying available requirement, and data replicas 
placement. In [1], authors proposed a data replication strategy to consider and optimize energy consumption, network bandwidth consumption and communication delay for geographically diversely-located data centers as well as inside each data center. In [15], authors proposed a cost-effective data replication strategy with a consideration of access frequency and the average response time to determine whether the dataset should be replicated or not in cloud environment. In [14], authors proposed a CDRM strategy as a cost-effective dynamic replication management scheme. They proposed a novel way to capture the relationship between availability and replica number. In [8], authors proposed two QoS-aware data replication (QADR) algorithms. The first algorithm is to perform data replication and the other one is to minimize the data replication cost and the number of QoS-violated data replicas.

Although many parameters have been considered to determine and constrain the data replication in related works. These parameters are used in an isolated way with some simple parameter combination, such as access frequency and average response time [15]. This may cause storage overloading problems in data centers. As a result, the existing approaches may only work in specific contexts. A more general data replication strategy that integrates various parameters and attributes needs to be investigated in order to comprehensively determine the data replication and further balance between the total cost and the situation in actual environment.

\section{SYSTEM MODEL}

\section{A. Dataset Classification}

In the previous literatures, many data placement strategies demonstrated that the dataset types are classified into two categories, fixed dataset and flexible dataset. A fixed dataset $(F D)$ cannot be replicated because of the constraints of its own attributes, for example, data ownership or privacy concerns, while a flexible dataset can be freely replicated among geographical data centers as well as inside data centers. This paper focus on the actual size constraints of data centers and further classify the flexible dataset into two novel dataset types, freeflexible dataset $(F F D)$ and constrained-flexible dataset $(C F D)$. These two novel dataset types $F F D$ and $C F D$ are corresponding to each individual data center in the cloud environment. A dataset $d_{i}$ can be classified to free-flexible dataset for data center $d c_{p}$ when the size of dataset $d_{i}$ less than current available storage capacity in the data center $d c_{p}$. On the other word, it means the flexible dataset $d_{i}$ is freely replicated when the dataset $d_{i}$ is a FFD for data center $d c_{p}$, Otherwise, the dataset $d_{i}$ should be constrained-flexible dataset for data center $d c_{p}$ when the dataset $d_{i}$ cannot meet the size constraints of data center $d c_{p}$.

\section{B. Data Storage Space Partition}

With the combination of practical environment, data centers cannot arrive their peak storage capacity value as normal. Thus, sometimes it may only be utilized within a range of percentage of the total storage capacity. We partition the storage space of data centers into two areas, initial and generated data storage space and replicated data storage space, which aims to constrain the data movement with the constraints between the size of replicated datasets and current available data storage capacity in replicated storage space. A parameter $\Omega$ is adopted to partition the storage space into two space, one is $S S_{\text {replicated }}=\Omega * S S_{\text {total }}$ for replicated data storage, and other is $S S_{\text {storage }}=S S_{\text {total }}-S S_{\text {replicated }}$ for initial data and generated data storage, where $S S_{\text {total }}$ denotes the total storage space of data centers. The parameter $\Omega$ is an environment-driven variable and depends on the setting by each data center. A dataset $d_{i}$ can be classified to free-flexible dataset for data center $d c_{p}$ when the size of dataset $d_{i}$ less than current available storage capacity within replicated storage space in the data center $d c_{p}$. It means the data replication should follow the size constraints as in (1), where ASS denotes the current Available Storage Space of the data center, and $A S S_{\text {replicated }}$ denotes available storage capacity within replicated data storage space [18].

$$
\text { Size }_{\text {replicated }} \leq A S S_{\text {replicated }}
$$

Along with increased data storage amounts within replicated data storage space $S S_{\text {replicated }}$, we can repeatedly calculate the current state of storage capacity within replicated data storage space $A S S_{\text {replicated }}=S S_{\text {replicated }}-S S_{\text {current-replicated }}$, where $S S_{\text {current-replicated denotes the storage }}$ capacity that is currently consumed by replicated data.

\section{Dataset Dependency and Access Frequency}

We calculate data dependencies between each two datasets and access frequencies of each dataset as two of the constraints of data replication. The data dependency is the relationship between each two datasets and the access frequency is the frequency of access in a specific time duration. The data dependency between each two datasets $d_{i}$ and $d_{j}$ is defined as the number of tasks that use both $d_{i}$ and $d_{j}$ [17]. Each data dependency $\operatorname{Dep}\left(d_{i}, d_{j}\right)$ can be calculated as in (2).

$$
\operatorname{Dep}\left(d_{i}, d_{j}\right)=\text { Count }\left(T\left(d_{i}\right) \cap T\left(d_{j}\right)\right)
$$

$T\left(d_{i}\right)$ denotes the number of tasks in $T$ which use the dataset $d_{i}$, and $T$ denotes the whole set of tasks in the workflow. Before the calculation of access frequency, we define the time interval of data transfer is same to that of data storage time $_{s}$ for simplicity of calculation and consistency of time interval. We calculate access frequencies for each datasets as in (3).

$$
\operatorname{Accf}_{d_{i}}=\text { AccNum }_{d_{i}} / \text { time }_{s}
$$


$A c c f_{d_{i}}$ denotes the access frequency of the dataset $d_{i}$, and $\operatorname{AccNum}_{d_{i}}$ denotes the number of access times of the dataset $d_{i}$.

\section{Cost Model}

For any dataset $d_{x}$, the total cost TCost is the sum of data storage cost $\operatorname{Cost}_{s}$ and the sum of data transfer cost Cost $_{t}$ as in (4).

$$
T \operatorname{Cost}=\sum \operatorname{Cost}_{\mathrm{s}}+\sum \operatorname{Cost}_{t}
$$

Data storage cost Cost $_{t}$ depends on several parameters such as the specific data storage price of the cloud service provider, the size of the dataset Size $\left(d_{x}\right)$, and the storage time length time $_{s}$. Data storage price of cloud service provider is often defined as $\gamma$ per unit per storage time unit. Therefore, data storage cost can be calculated as in (5).

$$
\text { Cost }_{s}=\gamma * \operatorname{Size}\left(d_{x}\right) * \text { time }_{s}
$$

Data transfer cost $\operatorname{Cost}_{t}$ depends on the transfer cost ratio $\alpha$ per unit, the size of the dataset Size $\left(d_{x}\right)$, and the access frequency of the dataset $d_{x}$, and unit of data transfer time interval which is equal to the storage time length time $_{s}$ in our calculation for simplicity and consistency. Therefore, data transfer cost can be defined as in (6).

$$
\text { Cost }_{t}=\alpha * \text { Size }\left(d_{x}\right) * \operatorname{Accf}_{d_{x}} * \text { time }_{s}
$$

Therefore, as mentioned above, the total cost can be defined as in (7).

TCost $=\sum$ Cost $_{s}+\sum$ Cost $_{t}=\sum \gamma *$ Size $\left(d_{x}\right) *$ time $_{s}+\sum \alpha *$ Size $\left(d_{x}\right) *$ Acc $_{d_{x}} *$ time $_{s}$

In our approach, we aims to save the total cost TCost by changing pre-set threshold parameters of the values of dataset dependency and access frequency, and pre-set partition parameters of partitioning storage space.

\section{DATA REPLiCATION STRATEGy AND AlgORITHM}

Our data replication strategy is a build-time data replication strategy. In our data replication strategy, we assume that initial data placement has been completed by a specific pre-existing data placement strategy. The initial data placement is shown in Figure 1. The set of datasets $D$ will be allocated in data centers $D C$ based on the rule of data placement strategy. Especially, the set of datasets $D$ can be firstly partition into two datasets type, fixed datasets or flexible datasets, based on its initial attributes. Then they can be distributed to set of data centers $D C$ in different locations in the cloud environment.

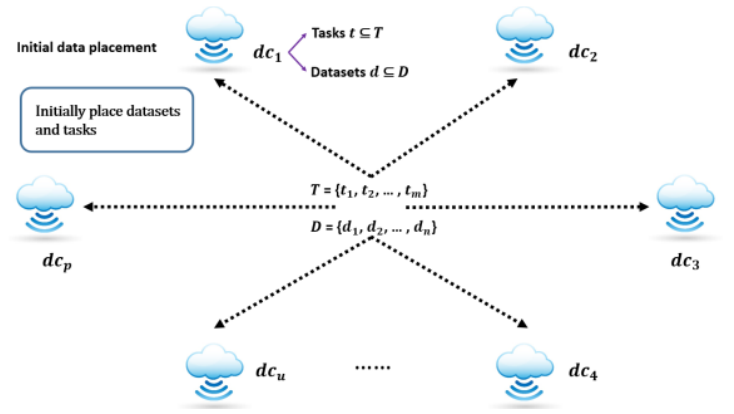

Fig. 1. Initial data placement

After all initial data placement finished, we start to locate the intersection of tasks $T\left(d_{i}\right) \cap T\left(d_{j}\right)$, which use both datasets $d_{i}$ and $d_{j}$, as shown in Figure 2 .

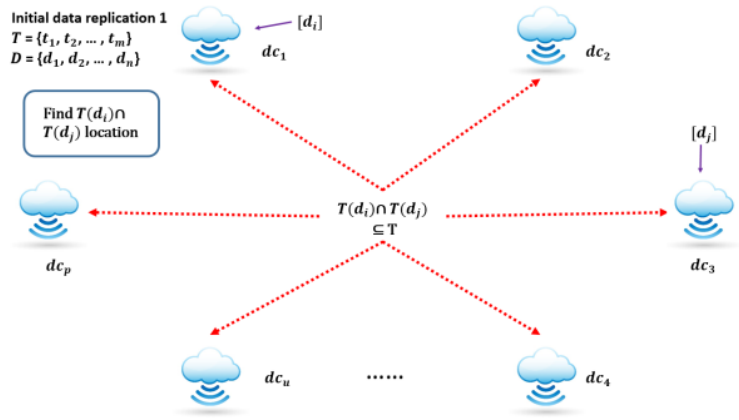

Fig. 2. Initial data replication 1

Then we start to determine the data replication for each two datasets. In this stage, dataset dependencies and access frequencies are calculated among set of datasets $D$. Then in our decision model, the mandatory requirement for data replication is $\operatorname{Dep}\left(d_{i}, d_{j}\right) \geq \beta$, where $\beta$ is the pre-set threshold parameter of the value of dataset dependency for constraining the dataset replication. Next, the access frequency of these datasets should be determined if either $A c c f_{d_{i}}$ or $A c c f_{d_{j}}$ satisfies $A c c f_{d_{i}} \geq \delta$ or $A c c f_{d_{j}} \geq \delta$, where $\delta$ is the pre-set threshold parameter of the value of access frequency. The dataset for replicating need satisfy both dataset dependency and access frequency constraints as the primary determinant level of data replication. In the secondary determinant level of data replication, eligible datasets are compared with each available storage space in geographical data centers where the intersection of task sets $T\left(d_{i}\right) \cap T\left(d_{j}\right)$ located. The primary and secondary determinant level decide the data replication together as shown in Figure 3 and 4.

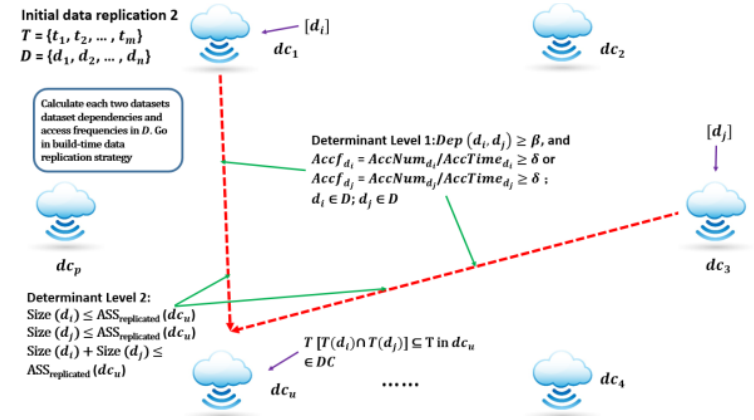

Fig. 3. Initial data replication 2 


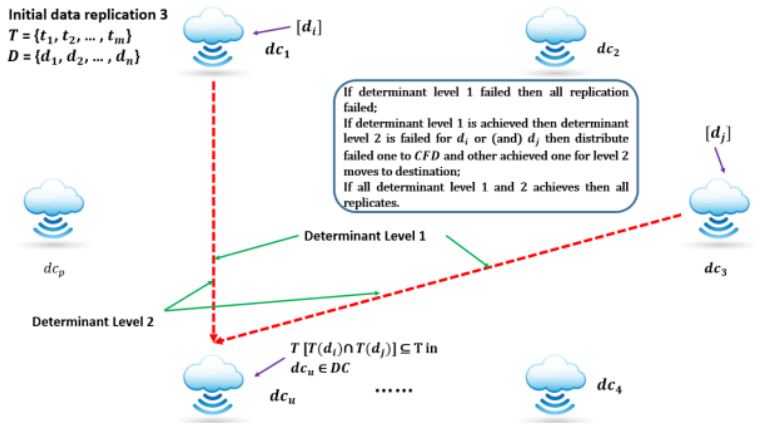

Fig. 4. Initial data placement 3

Our build-time data replication alogrithm is shown as follows.

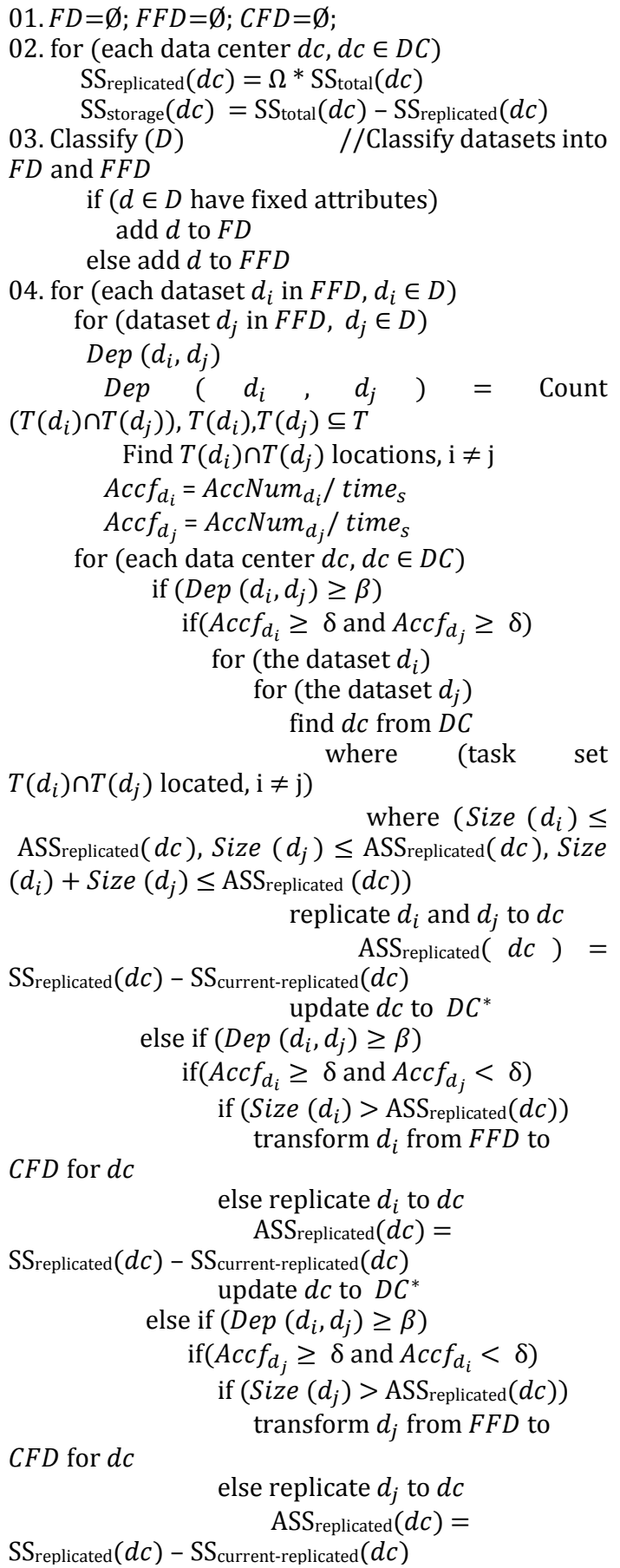

CFD for $d c$

else replicate $d_{i}$ to $d c$ $\operatorname{ASS}_{\text {replicated }}(d c)=$

SS replicated $(d c)-\mathrm{SS}_{\text {current-replicated }}(d c)$ update $d c$ to $D C^{*}$

else if $\left(\operatorname{Dep}\left(d_{i}, d_{j}\right) \geq \beta\right)$

$\operatorname{if}\left(A c c f_{d_{j}} \geq \delta\right.$ and $\left.A c c f_{d_{i}}<\delta\right)$

if $\left(\right.$ Size $\left.\left(d_{j}\right)>\mathrm{ASS}_{\text {replicated }}(d c)\right)$ transform $d_{j}$ from $F F D$ to

CFD for $d c$

else replicate $d_{j}$ to $d c$

$\operatorname{ASS}_{\text {replicated }}(d c)=$

$\mathrm{SS}_{\text {replicated }}(d c)-\mathrm{SS}_{\text {current-replicated }}(d c)$

FFD to $C F D$ for $d c$

update $d c$ to $D C^{*}$

else if $\left(\operatorname{Dep}\left(d_{i}, d_{j}\right) \geq \beta\right)$

$\operatorname{if}\left(A c c f_{d_{i}}<\delta\right.$ and $\left.A c c f_{d_{j}}<\delta\right)$

then transform $d_{i}$ and $d_{j}$ from

05. else (for each data center $d c, d c \in D C$ )

$$
\text { if }\left(\operatorname{Dep}\left(d_{i}, d_{j}\right)<\beta\right)
$$

06. Return $D C^{*}$

\section{CAse Study AND Discussion}

We use a sample workflow in [17] as a case study to compare the cost change with and without our data replication adoption. Sample workflow is shown in Figure 5 below.

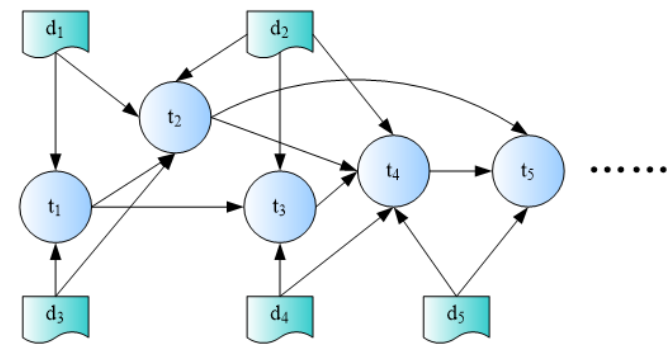

Fig. 5. Sample workflow

Firstly, we calculate dataset dependencies among each two datasets in this sample workflow based on dataset dependency model presented in Section III. The resultant data dependency values are stored in a data dependency matrix as shown in Figure 6 below.

$\begin{array}{cccccc} & d_{1} & d_{2} & d_{3} & d_{4} & d_{5} \\ d_{1} & 2 & 1 & 2 & 0 & 0 \\ d_{2} & 1 & 3 & 1 & 2 & 1 \\ d_{3} & 2 & 1 & 2 & 0 & 0 \\ d_{4} & 0 & 2 & 0 & 2 & 1 \\ d_{5} & 0 & 1 & 0 & 1 & 2\end{array}$

Fig. 6. Data dependency matrix

Therefore, if we set the threshold parameter of dataset dependency $\beta=1$, we can find that several dataset dependencies in this sample workflow meet the dataset dependency constraint $\operatorname{Dep}\left(d_{i}, d_{j}\right) \geq \beta$, for example $\operatorname{Dep}\left(d_{1}, d_{2}\right)$. We assume that the initial data placement in this sample workflow is already done by data placement strategy in [17], which is shown in Figure 7. 


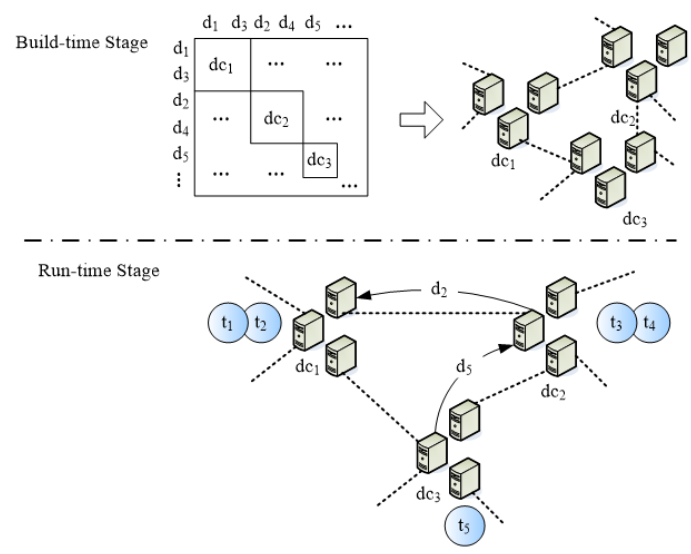

Fig. 7. Initial data placement in sample workflow

It shows that $\left(d_{1}, d_{3}\right)$ locate in $d c_{1},\left(d_{2}, d_{4}\right)$ locate in $d c_{2}$ and $\left(d_{5}\right)$ locate in $d c_{3}$. Therefore, based on our dataset dependency constraints, datasets $d_{2}$ and $d_{5}$ can be moved because $d_{2}$ located in $d c_{2}$ but should be invoked by task $t_{2}$ located in $d c_{1}$, similar to $d_{5}$. Therefore we propose to replicate $d_{2}$ and $d_{5}$ to $d c_{1}$ and $d c_{2}$ respectively. We assume that every data center size is large enough for data storage and processing in both replicated data storage space, and initial and generated data storage space. And then we assume the pre-set access frequency parameter $\delta$ is 0 to meet all access frequency constraints for each datasets in different data centers.

Without our data replication strategy, dataset $d_{2}$ is stored in $d c_{2}$. Every time it is accessed by task $t_{2}$ in $d c_{1}$, it needs to be transferred from $d c_{2}$ to $d c_{1}$ during task $t_{2}$ runs. This is similar situation to $d_{5}$.

To illustrate the effectiveness of our approach quantitatively, we use the dataset size value, data storage cost per unit per GB, data storage time and data transfer cost per unit per GB in Table I. The data storage and data transfer cost is based on and calculated from Microsoft Azure cloud storage services cost model in Australia East area.

TABLE I. THE VALUE OF PARAMETERS

\begin{tabular}{|c|c|}
\hline Parameters & Value \\
\hline time $_{s}$ & 1 month \\
\hline$\gamma$ & 0.175 per unit per GB \\
\hline$\alpha$ & $20 \mathrm{~GB}$ \\
\hline Size $\left(d_{2}\right)$ & $15 \mathrm{~GB}$ \\
\hline Size $\left(d_{5}\right)$ & 8 times $/$ month \\
\hline Acc $f_{d_{2}}$ & 10 times $/$ month \\
\hline$A c c f_{d_{5}}$ & \\
\hline
\end{tabular}

Apart from that, we assume that data storage time time $_{s}$ is only 1 month for simplicity. Finally, for the size of datasets $d_{2}$ and $d_{5}$ we assume they are in 20 GB and $15 \mathrm{~GB}$ respectively. Then, the access frequency of datasets $d_{2}$ and $d_{5}$ is assumed by us in 8 times per month and 10 times per month respectively.
Situation 1: we assume the workflow application processing in the cloud only exploits data placement strategy.

In this situation, dataset $d_{2}$ will be exploited 8 times during its processing stage. We ignore the cost of other datasets because they will fix in their initial data centers and have a constant total cost during all task processing stage. It means the cost of dataset $d_{2}$ is the sum of the data storage cost of dataset $d_{2}$ in $d c_{2}$ and the data transfer cost from $d c_{2}$ to $d c_{1}$. This situation is similar to $d_{5}$. Therefore, the total cost will be as follows:

$$
T \text { Cost }=\sum \operatorname{Cost}_{s}+\sum \operatorname{Cost}_{t}=59.76 \text { per unit per } G B
$$

Situation 2: we assume the workflow application processing in the cloud environment exploits our data replication strategy after initial data placement.

In this situation, dataset $d_{2}$ will be replicated to data center $d c_{1}$ before the run-time stage. We ignore the cost of other datasets because they will fix in the initial data center and have a constant total cost during all task processing stage, which is similar to situation 1. The cost of dataset $d_{2}$ is the sum of data storage cost for dataset $d_{2}$ in $d c_{2}$ and destination data center $d c_{1}$, and data transfer cost from $d c_{2}$ to $d c_{1}$ in one time based on our data replication strategy. This is similar to $d_{5}$. Therefore, the cost will be as follows:

TCost $=\sum$ Cost $_{s}+\sum$ Cost $_{t}=18.31$ per unit per $G B$

It is obviously shown that the total cost have a big percentage decrease after exploiting our data replication strategy to compare with the total cost of only data placement strategy exploitation. There are obvious $69.36 \%$ decrease from 59.76 per unit per $G B$ to 18.31 per unit per $G B$ on total cost after exploiting our data replication strategy as shown in Figure 8.

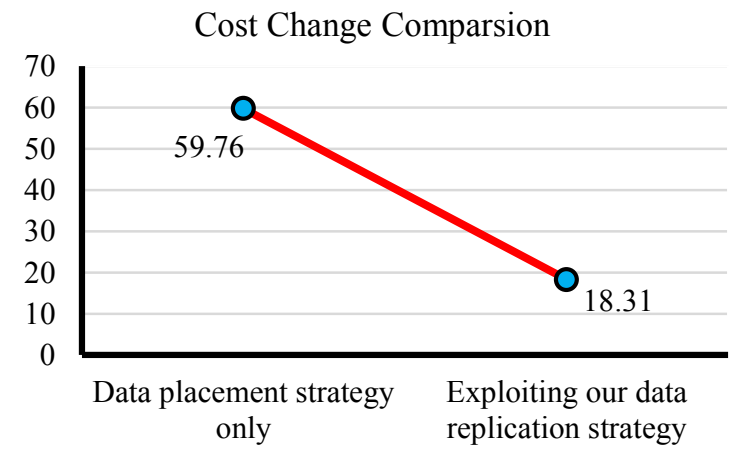

Fig. 8. Cost change comparison

As a result, we can find that our data replication strategy can significantly reduce the total cost of data management for cloud-based applications. In our approach, we set several values of parameters in the cloud environment for calculation simplicity. But our strategy can also implement in actual environment with different parameter values and workflow structures. The parameter value and the workflow structure are an important factor to influence the percentage of total cost reduction because our constraints of dataset dependency, access frequency, 
storage capacities of data centers, size of datasets are highly related to those parameter values and workflow structures.

\section{CONCLUSIONS AND FUTURE WORK}

In conclusions, data replication is commonly used to decrease access latency, improve data availability, and reduce data transfer cost by replicating data replicas to geographical data centers. In this paper, we propose a novel environment-driven data replication strategy for cloud-based workflow applications in a cost-effective view by considering the constraints of dataset dependency, access frequency, storage capacities of data centers, and size of datasets in the build-time stage. We use a case study to compare our data replication strategy adoption with only data placement strategy exploitation. The case study shows that our data replication strategy can greatly reduce the total cost of data management for workflow applications.

In the future, we will further consider run-time data replication strategy for workflow applications. We will also process the placement and replication of generated datasets to further reduce the total cost. Further simulations on complex workflows are also needed to evaluate our strategy under different scenarios in a cost-effective view.

\section{REFERENCES}

[1] Boru, D, Kliazovich, D, Granelli, F, Bouvry, P \& Zomaya, AY 2015, 'Energy-efficient data replication in cloud computing datacenters', Cluster Computing, vol. 18, no. 1, pp. 385-402.

[2] Boru, D, Kliazovich, D, Granelli, F, Bouvry, P \& Zomaya, AY 2015, 'Models for efficient data replication in cloud computing datacenters', in Communications (ICC), 2015 IEEE International Conference on, pp. 6056-6061.

[3] Gill, NK \& Singh, S 2014, 'Dynamic Cost-Aware Rereplication and Rebalancing Strategy in Cloud System', in FICTA (2), pp. 39-47.

[4] Janpet, J \& Wen, Y-F 2013, 'Reliable and available data replication planning for cloud storage', in Advanced Information Networking and Applications (AINA), 2013 IEEE 27th International Conference on, pp. 772-779.

[5] Khalajzadeh, H, Yuan, D, Grundy, J \& Yang, Y 2016, 'Improving Cloud-based Online Social Network Data Placement and Replication', in Cloud Computing (CLOUD), 2016 IEEE 9th International Conference on, pp. 678-685.

[6] Li, W, Yang, Y \& Yuan, D 2011, 'A novel cost-effective dynamic data replication strategy for reliability in cloud data centres', in Dependable, Autonomic and Secure Computing (DASC), 2011 IEEE Ninth International Conference on, pp. 496-502.

[7] Li, W, Yang, Y \& Yuan, D 2016, 'Ensuring Cloud data reliability with minimum replication by proactive replica checking', IEEE Transactions on Computers, vol. 65, no. 5, pp 1494-1506.

[8] Lin, J-W, Chen, C-H \& Chang, JM 2013, 'QoS-aware data replication for data-intensive applications in cloud computing systems', IEEE Transactions on Cloud Computing, vol. 1, no. 1, pp. 101-115.

[9] Liu, G, Shen, H \& Chandler, H 2016, 'Selective data replication for online social networks with distributed datacenters', IEEE Transactions on Parallel and Distributed Systems, vol. 27, no. 8, pp. 2377-2393.

[10] Long, S-Q, Zhao, Y-L \& Chen, W 2014, 'MORM: A Multiobjective Optimized Replication Management strategy for cloud storage cluster', Journal of Systems Architecture, vol. 60, no. 2, pp. 234-244

[11] Mansouri, N 2016, 'Adaptive data replication strategy in cloud computing for performance improvement', Frontiers of Computer Science, vol. 10, no. 5, pp. 925-935.

[12] Milani, BA \& Navimipour, NJ 2016, 'A comprehensive review of the data replication techniques in the cloud environments: Major trends and future directions', Journal of Network and Computer Applications, vol. 64, pp. 229-238.

[13] Wang, T, Yao, S, Xu, Z \& Jia, S 2016, 'DCCP: an effective data placement strategy for data-intensive computations in distributed cloud computing systems', The Journal of Supercomputing, vol. 72, no. 7, pp. 2537-2564.

[14] Wei, Q, Veeravalli, B, Gong, B, Zeng, L \& Feng, D 2010, 'CDRM: A cost-effective dynamic replication management scheme for cloud storage cluster', in Cluster Computing (CLUSTER), 2010 IEEE International Conference on, pp. 188196.

[15] Wu, X 2016, 'Data Sets Replicas Placements Strategy from Cost-Effective View in the Cloud', Scientific Programming, vol. 2016, doi: 10.1155/2016/1496714

[16] Ye, Z, Li, S \& Zhou, J 2014, 'A two-layer geo-cloud based dynamic replica creation strategy', Applied Mathematics \& Information Sciences, vol. 8, no. 1, pp. 431-439.

[17] Yuan, D, Yang, Y, Liu, X \& Chen, J 2010, 'A data placement strategy in scientific cloud workflows', Future Generation Computer Systems, vol. 26, no. 8, pp. 1200-1214.

[18] Zhao, Q, Xiong, C, Zhao, X, Yu, C \& Xiao, J 2015, 'A data placement strategy for data-intensive scientific workflows in cloud', in Cluster, Cloud and Grid Computing (CCGrid), 2015 15th IEEE/ACM International Symposium on, pp. 928-934. 